II. Fiktionen und Figuren 


\section{Wenn sich das Innere (nicht) im Äußeren spiegelt Physiognomik- und Pathognomik-Reflexion in Sophie von La Roches Geschichte des Fräuleins von Sternheim}

\section{Lavater und die Verbreitung physiognomischen Wissens}

Johann Caspar Lavater hat die Physiognomik nicht erfunden. Aber er hat sie in ausgesprochenem Maße popularisiert, gar zu einer Mode-Wissenschaft seiner Zeit avancieren lassen, die zeitweilig selbst so berühmte Anhänger wie Goethe gefunden hat. Wenige Jahre nach Drucklegung von Lavaters Schriften muss Georg Christoph Lichtenberg bereits konstatieren, dass die "Physiognomik Mode geworden ist ${ }^{1} .{ }^{1}$ Nicht nur verzeichnen die Zeitgenossen eine "schnelle Ausbreitung der Physiognomick «, ${ }^{2}$ sondern diagnostizieren den Ausbruch einer wahren "physiognomische[n] Wuth,$^{3}$ die alsbald heftigen Attacken ausgesetzt ist. ${ }^{4}$

Schon im zeitgenössischen Diskurs verknüpft sich dabei der Name Lavaters so eng mit der Physiognomik, dass er zu ihrem metonymischen Stellvertreter wird, ja im kollektiven Bewusstsein nicht allein den Anfangspunkt

1 [Georg Christoph Lichtenberg]: Ueber Physiognomik; wider die Physiognomen. Zu Beförderung der Menschenliebe und Menschenkenntniß. Zweyte vermehrte Aufl., Göttingen 1778, S. 67.

2 Ebd., S. 7.

3 Lexikon Deutscher Dichter und Prosaisten, hg. von Karl Heinrich Jördens, Bd. 3, Leipzig 1808, Lemma: Johann Kaspar Lavater, S. 155-231, hier S. 186.

4 Vgl. Georg Christoph Lichtenberg: Ueber Physiognomik; wider die Physiognomen. Angesichts der nicht abebbenden Physiognomikflut lässt Lichtenberg dem einige Jahre später die bissige Lavater-Parodie nachfolgen: [ders.]: Fragment von Schwänzen. Ein Beytrag zu den Physiognomischen Fragmenten, o.O. 1783. Während Lichtenberg die Lavater'sche Physiognomik einer wissenschaftsmethodologischen Kritik unterzieht, unternimmt beispielsweise Johann Karl August Musäus eine Überprüfung der physiognomischen Praxis im Sinne symbolischen Probehandelns - im Medium der Literatur: Johann Karl August Musäus]: Physiognomische Reisen voran ein physiognomisch Tagebuch. Heftweis' herausgegeben, 4 Bde., Altenburg 1778/79. Mögen auch die Darstellungen Lichtenbergs und Musäus' hyperbolischen Charakter tragen, so geben sie doch beredtes Zeugnis ab von der als rasant empfundenen Karriere der Lavater'schen Physiognomik und ihren Ausläufern, steht doch die Überdeutlichkeit von Überzeichnungen letztlich im rhetorischen Dienste der Verdeutlichung. 
der physiognomischen Mode, sondern der physiognomischen Praxis selbst zu markieren scheint. Dies lässt sich etwa an Balthasar Anton Dunkers Schriften aus dem Jahre 1782 ablesen, deren Titelblatt anstelle des Namens den Schattenriss des Autors zeigt. Zwar ist sich Dunker der Historizität der physiognomischen Tradition bewusst, wenn er hier in einer satirischen, mit seiner Silhouette signierten Dedikation die »Wiedereinsetzung « der »Göttin« Physiognomik auf ihren "alten Thron, der fast gar schon zusammenfallen wollte«, verzeichnet. ${ }^{5}$ Zugleich aber datiert Dunker seine der Physiognomik zugeeigneten Schriften, nicht ohne Augenzwinkern, mit der Bestimmung »im achten Jahre nach dem Einfall der Silhouetten in Deutschland $\aleph^{6}$ und lässt damit das Erscheinungsjahr des ersten Bandes von Lavaters Physiognomischen Fragmenten nicht allein als Ausgangspunkt der Physiognomik, sondern einer neuen Zeitrechnung schlechthin erscheinen.

Dabei findet sich die Physiognomik sowohl als Praxis als auch Reflexionsgegenstand schon vor den, durch die zahlreichen Kupferstiche ohnedies sehr teuren, zunächst nur für eine kleine Leserschaft erschwinglichen Physiognomischen Fragmenten. ${ }^{7}$ Die Physiognomik ist auch 'vor Lavater Teil des kulturellen Wissens der Epoche und wird von so unterschiedlichen Autoren wie etwa Christian Adam Peuschel ${ }^{8}$ oder Julius Bernhard von Rohr ${ }^{9}$ verbreitet. So

5 Alle Teilzitate: [Balthasar Anton Dunker]: Schriften von [Schattenriss], Bern 1782, Zueignungs-Schrift an die Physiognomik [unpag.].

6

7 "Aber dieses Werk ist sehr kostbar und prachtreich? Antwort: Es ist durchaus nicht für den großen Haufen geschrieben. Es soll von dem gemeinen Manne nicht gelesen und nicht gekauft werden. Es ist kostbar seiner Natur nach; kostbarer als andere Werke mit Kupfern, weil sehr viele Zeichnungen und Kupferplatten fehlgeschlagen sind - übrigens, können's verschiedene zusammenkaufen und gemeinschaftlich besitzen. - (Johann Caspar Lavater: Physiognomische Fragmente, zur Beförderung der Menschenkenntniß und Menschenliebe. Erster Versuch. Mit vielen Kupfern, Leipzig/Winterthur 1775, Vorrede [unpag.]) Erst in den Jahren 1783 bis 1787 erscheint eine billigere Auswahlausgabe.

8 Peuschel veröffentlicht im Jahre 1769 eine Abhandlung der Physiognomie, Metoposcopie und Chiromantie, worin das physiognomische Wissen der zurückliegenden Jahrhunderte versammelt und die physiognomische Deutung der Praxis der Weissagung, d.h. der zukunftsgerichteten Prophetie, zugeordnet wird - wofür er nicht zuletzt von Lavater Kritik ernten soll: Christian Adam Peuschel: Abhandlung der Physiognomie, Metoposcopie und Chiromantie, mit einer Vorrede, darinnen die Gewißheit der Weißagungen aus dem Gesichte, der Stirn und den Händen gründlich dargethan wird, welcher am Ende noch einige Betrachtungen und Anweisungen zu weißagen beygefügt worden, die zur bloßen Belustigung dienen, Leipzig 1769.

9 Julius Bernhard von Rohr: Unterricht Von der Kunst der Menschen Gemüther zu erforschen, Darinnen gezeiget, In wie weit man aus eines Reden, Actionen und anderer Leuten Urtheilen, eines Menschen Neigungen erforschen könne, Und überhaupt untersucht wird, 
wenig allerdings die Physiognomik eine Erfindung Lavaters ist, so wenig auch ist sie eine Erfindung des 18. Jahrhunderts. Sieht man einmal über einzelne konzeptuelle Differenzen hinweg, so lässt sich die Vorstellung, dass das sinnlich wahrnehmbare körperliche Äußere ein Zeichen für sinnlich nichtwahrnehmbare innere Eigenschaften sei, bereits auf das kalokagathia-Ideal der griechischen Antike zurückverfolgen, das körperliche und moralische, äußere und innere Schönheit in eins setzt. ${ }^{10}$

Wenn nun Sophie von La Roche ab dem Jahr 1766 ansetzt, ihren Briefroman Geschichte des Fräuleins von Sternheim zu schreiben, hat sie Lavaters Schriften noch nicht lesen und die dadurch katalysierte Physiognomik-Mode noch nicht erleben können. Die Geschichte des Fräuleins von Sternheim, die sich zu einem wahren Erfolgsbuch entwickeln und noch im ersten Jahr drei Auflagen erleben wird, erscheint nach fünfjähriger Arbeit im Jahre 1771. Lavaters Gedanken Von der Physiognomik ${ }^{11}$ folgen erst 1772, die Physiognomischen Fragmente, zur Beförderung der Menschenkenntnis und Menschenliebe ${ }^{12}$ in den Jahren 1775 bis 1778. Die Geschichte des Fräuleins von Sternheim liefert keinen vvorweggenommenen Kommentar zu den Schriften Lavaters, der sich im Übrigen zum Briefpartner und Gast in La Roches literarischem Salon entwickeln wird. Vielmehr unternimmt sie eine literarische Reflexion der Episteme der Zeit. Wenn im vorliegenden Beitrag, zeitlich vorausgreifend, dennoch immer wieder auf Lavater verwiesen wird, dann ist dies einzig der Intention geschuldet,

Was bey der gantzen Kunst wahr oder falsch, gewiß oder ungewiß sey, 3. Aufl., Leipzig 1721. Während die Erstausgabe des Jahres 1713 in Einlösung der Versprechungen des Titelblattes noch ausschließlich darzulegen bemüht ist, wie man »der Menschen Gemüther [...] aus eines Reden, Actionen und anderer Leuten Urtheilen [...] erforschen könne«, fügt von Rohr ab der dritten Auflage ein (nicht unkritisches) Kapitel »Von der Physiognomie» hinzu, setzt allerdings den ihm wohl etwas fremd erscheinenden Begriff der >Physiognomier bzw. Physiognomia typografisch in Antiqua ab.

10 Vgl. grundlegend Robert E. Norton: The Beautiful Soul. Aesthetic Morality in the Eighteenth Century, Ithaca/London 1995. Zur physiognomischen Tradition svor Lavater vgl. zudem Martin Porter: Windows of the Soul. Physiognomy in European Culture 1470-1780, Oxford 2005, sowie Hartmut Böhme: Der sprechende Leib. Die Semiotiken des Körpers am Ende des 18. Jahrhunderts und ihre hermetische Tradition, in: Transfigurationen des Körpers. Spuren der Gewalt in der Geschichte, hg. von Dietmar Kamper und Christoph Wulf, Frankfurt a.M. 1989, S. 144-181.

11 Auf eine anonym erfolgende, durch Johann Georg Zimmermann im Hannoverischen Magazin platzierte Vortragspublikation folgt noch im selben Jahr mit dem gleichen Titel: Johann Caspar Lavater: Von der Physiognomik, 2 Bde., Leipzig 1772.

12 Johann Caspar Lavater: Physiognomische Fragmente, 4 Bde., Leipzig/Winterthur 17751778 . 
vermittels einer vergleichenden Kontrastierung die Spezifika von La Roches Physiognomik-Reflexion deutlicher aufscheinen zu lassen.

\section{Von Schattenrissen und Buchstaben: Medien der Physiognomik und Pathognomik}

Bevor jedoch die avisierte Analyse der Physiognomik-Reflexion in Sophie von La Roches Geschichte des Fräuleins von Sternheim erfolgen kann, sind eingangs noch einige Bemerkungen zum Wechselverhältnis von Physiognomik und ästhetischer Darstellung einerseits, von Körpersemiotik und Visualität andererseits vorzuschalten: Die Physiognomik im engen wie im weiten Sinne des Begriffsgebrauchs, d.h. die anhand ‘fester Körperpartien auf die Deutung grundlegender Charakterdispositionen zielende Praxis der Physiognomik im engen Sinne und ihr Komplement, die von ‘beweglichen Körperpartien auf aktuale Affektregungen schließende Pathognomik, ${ }^{13}$ sind eng verknüpft mit dem Reich der Ästhetik. Nicht nur bildet die Pathognomik mit ihrem Interesse am Ausdrucksvermögen der Mimik und an Körperzeichen im Allgemeinen eine Grundlage der sich im 18. Jahrhundert ereignenden Neujustierung der Schauspielkunst, ${ }^{14}$ sondern ästhetische Darstellungen werden ihrerseits zu Studienobjekten, denen man mit physiognomischen und pathognomischen Methoden beizukommen sucht.

13 Lavater erläutert die Differenz zwischen der Physiognomik im engen Sinne und der Pathognomik, indem er die (Nicht-)>Beweglichkeit‘ von Körper und Charakter parallelführt: "Physiognomik, im eingeschränkten Sinne des Wortes, ist Kraftdeutung, oder Wissenschaft der Zeichen der Kräfte. I Pathognomik, Leidenschaftsdeutung, oder Wissenschaft der Zeichen der Leidenschaften. Jene zeigt den stehenden - diese den bewegten Charakter. / Der stehende Charakter liegt in der Form der festen, und in der Ruhe der beweglichen Theile. Der leidenschaftliche - in der Bewegung der beweglichen. [...] / Physiognomik zeigt die Summe der Capitalkraft - Pathognomik das Interesse, das jene abwirft. Jene, was der Mensch überhaupt ist; diese, was er in dem gegenwärtigen Moment ist. (Johann Caspar Lavater: Physiognomische Fragmente, Bd. 4, Leipzig/ Winterthur 1778, Erster Abschnitt, II. Fr., S. 39.) Im weiteren Sinne wird der Begriff der 'Physiognomik indes häufig als Hyperonym für beide Deutungspraktiken verwendet.

14 Vgl. exemplarisch Ruedi Graf: Utopie und Theater. Physiognomik, Pathognomik, Mimik und die Reform von Schauspielkunst und Drama im 18. Jahrhundert, in: Physiognomie und Pathognomie. Zur literarischen Darstellung von Individualität. Festschrift für Karl Pestalozzi zum 65. Geburtstag, hg. von Wolfram Groddeck und Ulrich Stadler, Berlin/ New York 1994, S. 16-33, sowie Giovanni Gurisatti: Die Beredsamkeit des Körpers. Lessing und Lichtenberg über die Physiognomik des Schauspielers, in: DVjs 67/3 (1993), S. 393-416. 
Das Ausdrucksvermögen des menschlichen Körpers und insbesondere des Gesichts, der Physiognomie, ${ }^{15}$ liegt in seinem semiotischen Wert als natürliches Zeichen begründet. So spricht denn Lavater von einer "unwillkührlichen Natursprache im Antlitze, und dem ganzen Aeußerlichen des Menschen «. ${ }^{16}$ Gleichwohl werden physiognomisch-pathognomische Deutungsoperationen nicht allein an lebendigen, sich der Betrachtung live darbietenden oder sich gar in (eo ipso ephemerer) Bewegung befindlichen Körpern vorgenommen, sondern auffallend häufig an Kunstwerken - gerade der bildenden Künste. ${ }^{17}$ Nur zu aufschlussreich sind die Titelkupfer des ersten und des vierten Bandes der Physiognomischen Fragmente, die in einer mise en abyme-Struktur bildliche Darstellungen bildlicher Darstellungen bilden.

Wiewohl ästhetische Darstellungen insbesondere der bildenden Künste ein privilegiertes Medium der Physiognomik darstellen, fungieren materielle Bilder und ihre visuell sichtbaren Elemente bemerkenswert selten als ausschließlicher Gegenstand der Physiognomik, ist die Lesbarkeit von Körpern und die Evidenzkraft von Bildern nicht strikt an die Visualität gebunden. Im Gegenteil: Charakterologische respektive kulturelle Vorwissensbestände in Verbund mit der Einbildungskraft als der Fähigkeit, »eine sinnliche Vorstellung von einer abwesenden Sache 18 aufscheinen zu lassen, können als assoziatives Sprungbrett oder auch Korrektiv dienen. Nicht zuletzt die Problematik, dass »die verschiedenen Vorstellungsarten der Mahler, und ihre verschiedenen Fähigkeiten ein und ebendenselben Mann umbilden und verschieben « ${ }^{19}$ können, führt zu der Notwendigkeit, zum Gegenstand und mithin zur ontologischen Grundlage der Physiognomik, nicht ausschließlich das realiter sichtbare, materielle Bild zu bestimmen, sondern dieses durch ein mentales `Bild zu ergänzen oder auch zu ersetzen, das seinerseits freilich per

15 Bezeichnenderweise unterscheiden sich die `Physiognomik in einem Buchstaben.

16 Johann Caspar Lavater: Physiognomische Fragmente, Bd. 1, Leipzig/Winterthur 1775, Vorrede [unpag.].

17 Für Lavaters Physiognomik, die sich dezidiert als eine Physiognomik im engen Sinne verstanden wissen will und sich der charakterologischen Ausdeutung der sogenannten 'festen Körperpartien widmet, ist eine Stillstellung des Körpers im Abbild geradezu methodologisches Gebot. Nicht zufällig weisen Lavaters physiognomische Arbeiten eine hohe Affinität zur Kulturtechnik des Schattenrisses auf, die Gesichtszüge auf die Linie ihrer Silhouette reduziert.

18 Johann Christoph Adelung: Grammatisch-kritisches Wörterbuch der Hochdeutschen Mundart, Bd. 1, Leipzig 1793, Lemma: Die Einbildungskraft, S. 1689.

19 Johann Caspar Lavater: Physiognomische Fragmente, Bd. 2, Leipzig/Winterthur 1776, XXXIV. Fr., 9. und 10. Taf., S. 276. 
definitionem jenseits jeglicher visueller Wahrnehmbarkeit liegt. Selbst Lavater, der die Physiognomik insgesamt als eine Augen-Wissenschaft begreift (deren Zeichen »jedes gesunde Auge [...] wird finden und erkennen können $\left.{ }^{20}\right)$, kommt bei aller Konzentration auf visuelle Darstellungen nicht umhin, darauf hinzuweisen, dass ein Schattenriss bisweilen "um etwas verschnitten" oder ein Bild »verzeichnet« sei. ${ }^{21}$

Trotz aller Unzulänglichkeiten der bildenden Künste werden diese gegenüber sprachlichen Darstellungen aber stets bevorzugt, wann immer es um physiognomisch-pathognomische Erkenntnisgenerierung, Wissensfixierung und -vermittlung geht. ${ }^{22}$ So lässt sich Lavater zufolge die "unwillkührliche Natursprache" der menschlichen Physis "am natürlichsten" durch die "Sprache" der Bildkünste erfassen und studieren. Zur Zeichnung heißt es beispielsweise:

Zeichnung ist die erste, die natürlichste, die sicherste Sprache der Physiognomik; das beste Hülfsmittel für die Imagination; das einzige Mittel unzählige Merkmale, Ausdrücke und Nüances zu sichern, zu bezeichnen, mittheilbar zu machen, die nicht mit Worten, die sonst auf keine Weise zu beschreiben sind. Der Physiognomist, der nicht zeichnen kann, schnell, richtig, bestimmt, characteristisch zeichnen wird unzählige Beobachtungen nicht einmal zu machen, geschweige zu behalten und mitzutheilen, im Stande seyn. ${ }^{23}$

Die gestaltgebenden Künste aber werden nicht nur gegenüber der Sprache und ihren arbiträren Zeichen, sondern auch gegenüber der Natur selbst als bevorzugtes Medium der Physiognomik und Pathognomik inthronisiert, sind keineswegs 'nur Supplement sprachlicher Darstellungen, sondern in paradoxer Weise natürlicher noch als die Natur. So lässt sich Sulzers Allgemeiner Theorie der Schönen Künste entnehmen,

daß jedes vollkommene Portrait ein wichtiges Gemählde sey, weil es uns eine menschliche Seele von eigenem persönlichen Charakter zu erkennen giebt. Wir

20 Ders.: Physiognomische Fragmente, Bd. 1, Leipzig/Winterthur 1775, Vorrede, [unpag.].

21 Zum Einfluss der Hand des Künstlers auf die physiognomische Darstellung vgl. den Beitrag von Hans-Georg von Arburg in diesem Band.

22 Zur eschatologisch-sprachutopischen Dimension des Bildeinsatzes und Lavaters Suche nach einer adamitischen Ursprache vgl. Charlotte Kurbjuhn: Kontur. Geschichte einer ästhetischen Denkfigur, Berlin/Boston 2014, S. 319-335. Zur Problematik von Schrift, Bild und Evidenz bei Lavater vgl. Ursula Geitner: Klartext. Zur Physiognomik Johann Caspar Lavaters, in: Geschichten der Physiognomik. Text, Bild, Wissen, hg. von Rüdiger Campe und Manfred Schneider, Freiburg i.Br. 1996, S. 357-385.

23 Johann Caspar Lavater: Physiognomische Fragmente, Bd. 1, XV. Fr., S. 175 [meine Hervorhebung, Y.M.]. 
sehen in demselben ein Wesen, in welchem Verstand, Neigungen, Gesinnungen, Leidenschaften, gute und schlimme Eigenschaften des Geistes und des Herzens auf eine ihm eigene und besondere Art gemischt sind. Dieses sehen wir so gar im Portrait meistentheils besser, als in der Natur selbst; weil hier nichts beständig, sondern schnell vorübergehend und abwechselnd ist: $\mathrm{Zu}$ geschweigen daß wir selten in der Natur die Gesichter in dem vortheilhaften Lichte sehen, in welches der geschickte Mahler es gestellt hat. ${ }^{24}$

Wenn nun Sophie von La Roche in ihrem Briefroman Geschichte des Fräuleins von Sternheim trotz des Primats der Gestaltkünste auf dem Gebiet der Physiognomik resp. Pathognomik nicht nur (dabei freilich gattungskonform) ohne Abbildungen jeglicher Art auskommt, sondern auch gänzlich auf physische Detailbeschreibungen verzichtet, dann ist dies nicht allein den Transpositionsschwierigkeiten der visuell wahrnehmbaren Physis in das Zeichensystem der Schrift geschuldet, sondern schlicht und ergreifend einem gänzlich anderen Reflexionsfokus. Zur Verhandlung steht nicht die exakte Erfassung des Äußeren, sondern die Problematik des Schlusses vom Äußeren auf das Innere, mithin die mentale Operation selbst - so die These des vorliegenden Beitrags, der La Roches Briefroman als kulturelles Reflexionsmedium liest. Tatsächlich unterlässt der Roman nicht nur den Versuch, die fehlende Visualität von Körpern sprachlich zu ’kompensieren<, sondern thematisiert - ganz im Gegenteil - die Perspektivgebundenheit und Interpretationsabhängigkeit von Wahrnehmungen und die Grenzen einer Konzentration auf sichtbare Körper-Oberflächen.

Wie im Folgenden gezeigt werden soll, führt La Roches Geschichte des Fräuleins von Sternheim systematisch die Fehleranfälligkeit physiognomisch-pathognomischer Zeichendeutungen vor. Während Lavater in seiner Physiognomik wenig später einen absoluten Wahrheitsanspruch formulieren und »die innere objectivische Zuverläßigkeit der Physiognomieen « ${ }^{25}$ postulieren wird, ${ }^{26}$ erfolgen in der Geschichte des Fräuleins von Sternheim gerade deshalb Fehldeu-

24 Johann Georg Sulzer: Allgemeine Theorie der schönen Künste in einzeln, nach alphabetischer Ordnung der Kunstwörter auf einander folgenden, Artikeln abgehandelt, Bd. 2, Leipzig 1774, Lemma: Portrait, S. 918-921, hier S. 919 [meine Hervorhebung, Y.M.]. Lavater zitiert diese Passage; vgl. Johann Caspar Lavater: Physiognomische Fragmente, Bd. 2, IX. Fr., S. 80f.

25 Ders.: Physiognomische Fragmente, Bd. 1, Leipzig/Winterthur 1775, VII. Fr., S. 44.

26 Wie es Ursula Geitner formuliert, ist die Lavater'sche Physiognomik letztlich ein "Traum vom Verstehen ohne Rest“ (Ursula Geitner: Die Sprache der Verstellung. Studien zum rhetorischen und anthropologischen Wissen im 17. und 18. Jahrhundert, Tübingen 1992, S. 239). 
tungen, weil die menschlichen Gesichtszüge als natürliche Zeichen in ihrer Allgemeingültigkeit und Unfehlbarkeit nicht in Zweifel gezogen werden. Anders ausgedrückt: La Roches Figuren irren, weil sie das unsichere ${ }^{27}$ Wissen der Physiognomik als ein sicheres behandeln.

\section{La Roche: Die Grenzen physiognomischer und pathognomischer Zeichenlektüren}

In La Roches Briefroman setzt der libertin Lord Derby seine Beobachtungsgabe strategisch zur Verführung des Fräuleins von Sternheim ein. ${ }^{28}$ Derby fasst sein anthropologisches Wissen konzise zusammen, wenn er definiert, "daß unser Gesicht, und das was man Physionomie nennt, ganz eigentlich der Ausdruck unsrer Seele ist «. ${ }^{29}$ Nicht nur bezeichnet Derby damit eine unmittelbare Relation von außen und innen, von Signifikant und Signifikat, sondern zugleich das Paradoxon der Deutungstechniken 'Physiognomik und `Pathognomik`, dass im Äußeren des Menschen »ein Inneres [erscheint], das nur insofern wahrnehmbar ist, als es ein Äußeres ist, d.h. insofern es sich unmittelbar als sichtbar darbietet, obwohl es unsichtbar bleibt«.$^{30}$ Oder anders und mit Lavater ausgedrückt, wird hier die der Wahrnehmung zugängliche Physiognomie als "wahrer sichtbarer Ausdruck innerer an sich selbst unsichtbarer Eigenschaften « ${ }^{31}$ gefasst.

Dabei legt Derby die Visualität als Vorbedingung der gestaltdeutenden Kulturtechnik der Physiognomik respektive Pathognomik in durchaus engem Sinne aus und sieht allein das Auge als Organ der physiognomisch-patho-

27 Selbst Sulzer kann - seinem eigenen Glauben an die Physiognomik zum Trotz - nicht umhin, zu beobachten, "[w]ie ungegründet den meisten Menschen die Physiognomik, oder die Wissenschaft aus dem Gesicht und der Gestalt des Menschen seinen Charakter zu erkennen, vorkommen mag“ (Johann Georg Sulzer: Allgemeine Theorie der schönen Künste, S. 918).

28 Zum Verführungstopos vgl. Anna Marx: Das Begehren der Unschuld. Zum Topos der Verführung im bürgerlichen Trauerspiel und (Brief-)Roman des späten 18. Jahrhunderts, Freiburg i.Br. 1999.

29 [Sophie von La Roche]: Geschichte des Fräuleins von Sternheim. Von einer Freundin derselben aus Original-Papieren und andern zuverläßigen Quellen gezogen. Herausgegeben von C.M. Wieland, 2 Bde., Leipzig 1771, hier Bd. 1, S. 340.

30 Giovanni Gurisatti: Die Beredsamkeit des Körpers, S. 393.

31 Johann Caspar Lavater: Physiognomische Fragmente, Bd. 1, VII. Fr., S. 44 [meine Hervorhebung, Y.M.]. Entsprechend heißt es bei Sulzer: »[D]er Körper sey das Bild der Seele, oder die Seele selbst, sichtbar gemacht« (Johann Georg Sulzer: Allgemeine Theorie der schönen Künste, S. 918). 
gnomischen Wissensgenerierung an; er selbst ist nicht zufällig mit einem »feurige[n] Falkenauge « 32 ausgestattet. In welchem Maße die Ausdrucksfähigkeit des Gesichts an seine vollständige Sichtbarkeit gebunden ist, reflektiert Derby präzise, als er das Fräulein von Sternheim auf einem Maskenball beobachtet:

[O]hne Masque war meine Sternheim allezeit das Bild der sittlichen Schönheit, indem ihre Miene und der Blick ihrer Augen, eine Hoheit und Reinigkeit der Seele über ihre ganze Person auszugießen schien, wodurch alle Begierden, die sie einflößte, in den Schranken der Ehrerbietung gehalten wurden. Aber nun waren ihre Augenbraunen, Schläfe und halbe Backen gedeckt, und ihre Seele gleichsam unsichtbar gemacht; sie verlohr dadurch die sittliche charakteristische Züge ihrer Annehmlichkeiten und sank zu der allgemeinen Idee eines Mädchens herab. ${ }^{33}$

Die Konzentration auf das Visuelle, auf das, was man überhaupt sehen kann, erweist sich indes als epistemologische Falle. Diese Erfahrung muss, wiewohl in anderer Spielart, auch Fräulein von Sternheim machen: Wenn es ihn auch größte Mühe kostet, seine Leidenschaft »im Zügel zu halten«, 34 so ist doch Lord Derby dazu in der Lage, sein Äußeres daran zu hindern, sich als für das Fräulein von Sternheim sicht- und entzifferbare Projektionsfläche seines Inneren darzubieten (sein »äußerliches Ansehen sagte nichts davon ${ }^{35}$ ). Derby verkörpert das aus Sicht des 18. Jahrhunderts als ’höfisch<, rrhetorisch ${ }^{36}$ und (im moralischen Sinne) sunaufrichtig in Verruf geratene Paradigma einer privatpolitisch-kalkulierten Indienstnahme der Pathognomik als Kommunikationscode, in deren Zuge die eigene Körperoberfläche durch Verstellung unlesbar, die der anderen hingegen durchschaubar werden soll. Im Unterschied zum Fräulein von Sternheim begreift und praktiziert Derby die eloquentia corporis mithin als galantes Spiel von Oberflächencodes, nicht als Ausdrucksmedium wahrhaftiger Subjektivität. In diesem Spiel Derby versteht die Ereignisse bezeichnenderweise als Hofkomödie - geben die zuvorderst pathognomisch gewonnenen Erkenntnisse die nach außen zu spielende Rolle vor und dienen in umfassender Weise der Auswahl der Mittel, um das Fräulein von Sternheim zu verführen.

32 Sophie von La Roche: Geschichte des Fräuleins von Sternheim, S. 131.

33 Ebd., S. 340.

34 Ebd., S. 237.

35 Ebd., S. 247.

36 Vgl. grundlegend Ursula Geitner: Die Sprache der Verstellung, sowie Rüdiger Campe: Affekt und Ausdruck. Zur Umwandlung der literarischen Rede im 17. und 18. Jahrhundert, Tübingen 1990. 
Tatsächlich unterscheiden sich die Physiognomik im engen Sinne und die Deutungstechnik der Pathognomik nicht allein hinsichtlich ihres Beobachtungsgegenstandes (`fester vs. ‘beweglicher Körperpartien) und ihres Aussagegehalts (Charakterdispositionen vs. Affektregungen), sondern stellen verschiedene semiotische Verfahrensweisen dar, die in jeweils unterschiedlichen diskursiven Funktionszusammenhängen stehen. Die nicht erst durch Lavater geprägte begriffliche Unterscheidung zwischen der Physiognomik und der Pathognomik weist ins Zentrum des Verstellungsdiskurses. Während pathognomische Zeichen zum Verfolg einer kommunikativen Absicht bewusst produziert werden können, gelten physiognomische Zeichen als unwillkürlich und nichtmanipulierbar, stellen, mit Lavater gesprochen, eben eine »unwillkührliche[] Natursprache« dar. Während physiognomische Zeichen sich zeigen, werden pathognomische Zeichen gezeigt. Ein konstitutives Charakteristikum physiognomischer Zeichen ist, dass sie "nicht gemacht, sondern unabsichtlich hinterlassen [werden]« ${ }^{37}$ Erst dadurch avancieren sie zum Widerpart der unter den Generalverdacht der adeligen Galanterie und Unaufrichtigkeit gestellten Pathognomik, die sie im Zuge der Entrhetorisierung der Epoche allmählich ablösen. "Pathognomik hat mit der Verstellungskunst zu kämpfen; nicht so die Physiognomik «, ${ }^{38}$ schreibt Lavater, der betont, dass im Falle physiognomischer Zeichen "nicht die mindeste Verstellung Statt hat«.39

La Roches Briefroman ist von einem Netz aus Blicken durchzogen, ist Schauplatz einer literarischen Epistemologie, die sich in eminenter Weise für Blickregimes interessiert, für physiognomisch-pathognomische Selbstund Fremdbeobachtungen. ${ }^{40}$ Derby weiß genau: »Wachsam muß ich

37 Sibylle Krämer: Was also ist eine Spur? Und worin besteht ihre epistemologische Rolle? Eine Bestandsaufnahme, in: Spur. Spurenlesen als Orientierungstechnik und Wissenskunst, hg. von ders., Werner Kogge und Gernot Grube, Frankfurt a.M. 2007, S. 11-33, hier S. 16. Während physiognomische Zeichen die Eigenschaft der »Unmotiviertheit« mit Spuren teilen, ist ihnen hingegen das Moment des "Zeitenbruchs" (ebd., S. 17) nicht eigen.

38 Johann Caspar Lavater: Physiognomische Fragmente, Bd. 4, Erster Abschnitt, II. Fr., S. 39.

39 Ders.: Physiognomische Fragmente, Bd. 2, VII. Fr., S. 55.

40 So auch schon Alexandra Kleihues: „Die Geschichte des Fräulein von Sternheim stellt im wörtlichen Sinne ein Schau-Spiel dar: sämtliche Interaktionsverhältnisse werden im wesentlichen visuell hergestellt. Das Netz, in dem sich die Protagonistin nach und nach verfängt, wird gleichsam aus Blicken geknüpft [...]." (Alexandra Kleihues: Der empfindsame Blick. Zu Sophie von La Roches Geschichte des Fräuleins von Sternheim, in: Schaulust. Heimliche und verpönte Blicke in Literatur und Kunst, hg. von Ulrich Stadler und Karl Wagner, München 2005, S. 39-54, hier S. 41) 
seyn $[\ldots] .{ }^{41}$ Denn es gilt nicht nur, das Fräulein von Sternheim als »den Argus der Klugheit und Tugend einzuschläfern «, ${ }^{42}$ d.h. durch stete Aufmerksamkeit ihre Achtsamkeit auszuhebeln, sondern Derby hat unter aller Augen zu agieren, ist doch die höfische Kultur eine Kultur des Blicks und der Oberfläche, in der man »nur für Aug und Ohr lebt« ${ }^{43}$ Auf das Auge als Organ und Medium der Erkenntnis aber, so führt La Roches Briefroman verschiedentlich vor, ist nicht uneingeschränkt Verlass. Dies ist allerdings nicht viel mehr als ein locus communis. Lord Derby ist eine unter vielen Intrigantenfiguren, die mit ihren Verstellungskünsten die Literatur der Epoche bevölkern. Die 'Ratgeberliteratur des 16. und 17. Jahrhunderts hatte den Umstand, dass sich selbst die natürlichen Zeichen der Gesichtszüge verfälschen lassen, ja, dass speziell pathognomische Deutungen stets Gefahr laufen, einem bewusst herbeigeführten Hiatus zwischen Signifikat und Signifikant aufzusitzen, gerade zum Anlass genommen, eine praktische Menschenkenntnis als Teil der Lebenskunst zu vermitteln. ${ }^{44}$ Lavaters Fokusverlagerung auf die Physiognomik im engen Sinne ist nicht zuletzt dieser Problematik geschuldet.

Lord Derbys »feurige[m] Falkenauge« korrespondiert die Charakterisierung des Fräuleins von Sternheim als »Argus der Klugheit und Tugend «. ${ }^{45}$ Den Werte- und Verhaltenskodex der Aufrichtigkeit aus ihrem Elternhaus mitbringend, durchschaut Fräulein von Sternheim die höfische Welt durchaus als eine Kultur des Scheins und klagt - man beachte die Metaphorik des Sehvermögens in Bezug auf Attribute des Inneren -, "wie ermüdet« ihr "moralisches Auge über den täglichen Anblick des Erkünstelten im Verstande, in den Empfindungen, Vergnügungen und Tugenden ist $\aleph^{46}$ Bemerkenswer-

41 Sophie von La Roche: Geschichte des Fräuleins von Sternheim, S. $166 f$.

42 Ebd., S. 165 [meine Hervorhebung, Y.M.].

43 Ebd., S. 181. Zur Fundierung des höfischen Kommunikationssystems auf Veräußerlichung vgl. Albrecht Koschorke: Körperströme und Schriftverkehr. Mediologie des 18. Jahrhunderts, 2. durchges. Aufl., München 2003.

44 Paradigmatisch: Christian Thomasius: Erfindung der Wissenschaft anderer Menschen Gemüt zu erkennen, Schreiben an Friedrich III., Kurfürst von Brandenburg zu Neujahr 1692, in: Aus der Frühzeit der deutschen Aufklärung. Christian Thomasius und Christian Weise. Nachdruck der Ausgabe von 1928, hg. von Fritz Brüggemann, Darmstadt 1966, S. 61-79.

45 Sophie von La Roche: Geschichte des Fräuleins von Sternheim, S. 131, S. 165. Zu den religiösen (pietistischen) Vorzeichen des Beobachtens in La Roches Werk vgl. Ursula Naumann: Das Fräulein und die Blicke. Eine Betrachtung über Sophie von La Roche, in: Zeitschrift für deutsche Philologie 107 (1988), S. 488-516, bes. S. 501.

46 Sophie von La Roche: Geschichte des Fräuleins von Sternheim, S. 327 [meine Hervorhebung, Y.M.]. 
terweise jedoch versagt ihre Diskriminationsfähigkeit genau dann, wann immer sich etwas den Anschein des Tugendhaften gibt. Wie sich Fräulein von Sternheim von Derbys Beherrschung seiner Gesichtszüge täuschen lässt, so lässt sie sich auch von seiner Sprache in die Irre leiten, deren Oberfläche ihren eigenen Sprachgebrauch kopiert. Tatsächlich lässt sie sich bereits von einem schlagwortartigen Gebrauch des Wortes »Tugend« überzeugen, ohne zu argwöhnen, dass die »Tugend « - in Derbys Mund - nicht mehr als ein referenzloser Signifikant ist. Von einer Oberfläche täuschen lässt sich das Fräulein von Sternheim auch im Falle eines bukolischen Festes. Zwar erkennt sie durchaus scharfsinnig, dass im Landfest "[1]ändliche Einfalt und feine Hofkünste [...] sich so artig mit einander verwebt [fanden], daß man sie nicht trennen konnte «. ${ }^{47}$ Das Fräulein von Sternheim verkennt jedoch, dass diese inszenierte Ästhetik der Natürlichkeit sich einzig einem höfischen Komplott verdankt, das sie zur Mätresse des Fürsten zu machen sucht.

Während also das Fräulein von Sternheim nicht nur im Falle pathognomischer Deutungen an (handlungs-)entscheidenden Stellen von einer ungebrochenen Relation von Signifikat und Signifikant ausgeht und der sich darbietenden Oberfläche interpretatorisches Vertrauen schenkt, mithin ganz wie von vom Vater befürchtet, durch eine "Scheintugend hingerissen « 48 wird, beherrscht Derby die Praktiken sowohl der Verstellung als auch der Beobachtung virtuos. Doch selbst ihm unterlaufen im Verlauf der Geschichte des Fräuleins von Sternheim systematisch Fehldeutungen. Wie sehr Derby, der sich seiner »Kenntnisse des weiblichen Herzens «9 $^{4}$ brüstet, gerade bei der pathognomischen Deutung von Fräulein von Sternheims Mimik irrt, zeigt sich in der polyperspektivischen Anlage des Briefromans besonders merklich. Ein Beispiel: Bei einem Spaziergang gibt sich Fräulein von Sternheim glücklichen Erinnerungen, Gedanken an die Natur usw. hin. Sie selbst ist sich dabei durchaus bewusst, dass man ihr ihre Empfindungen ansehen (und anhören) kann: "Sie wissen [...][,] daß mein Gesicht allezeit die Empfindungen meiner Seele ausdrückt. Ich mag zärtlich und gerührt ausgesehen haben; der Ton meiner Stimme stimmte zu diesen Zügen. $\aleph^{50}$ Derby hingegen

47 Ebd., S. 259. Dies wird schon beobachtet in: Alexandra Kleihues: Der empfindsame Blick, S. 45f. Ferner unterlaufen Fräulein von Sternheim interpretatorische Fehler, weil sie die Inszenierung von Zufällen nicht als eine höfische Kunst erkennt und in ihrer Anglophilie alles Englische mit Tugendhaftigkeit engführt. Sophie von La Roche: Geschichte des Fräuleins von Sternheim, S. 71. Ebd., Bd. 2, S. 198.

50 Ebd., Bd. 1, S. 195. Darstellungslogisch führt die Ich-Perspektive der jeweiligen Briefe zur Paradoxie, sich selbst bisweilen von außen beschreiben zu müssen. 
missdeutet dies nun ausgerechnet als Zeichen der "Sehnsucht und Liebe» zu einem anderen Mann:

Das bezaubernde Fräulein von Sternheim heftete ihre Blicke auf eine gewisse Gegend; eine feine Röthe überzog ihr Gesicht und ihre Brust, die von der Empfindung des Vergnügens eine schnellere Bewegung zu erhalten schien. Sehnsucht war in ihrem Gesicht verbreitet, und eine Minute darauf stund eine Thräne in ihren Augen. [...] [A]lles [,] was ich jemals reizendes an andern ihres Geschlechts gesehen, ist nichts gegen den einnehmenden Ausdruck von Empfindung, der über ihre ganze Person ausgegossen war. [...] Rasend dachte ich, Sehnsucht und Liebe in ihr gesehen zu haben, die einen Abwesenden zum Gegenstand hatten [...]. ${ }^{51}$

Obwohl also die Figuren nie in Zweifel ziehen, dass die von ihnen beobachteten Körper etwas ausdrücken, erweist sich das Äußere des Menschen als ein polysemes Zeichen, das ganz unterschiedlich gedeutet werden kann. Wenn Lord Derby trotz seines "Falkenauges" im Handlungsverlauf systematisch Fehlschlüsse zu erleiden hat, dann liegt dies nicht zuletzt daran, dass das semiotische System, anhand und innerhalb dessen er physiognomisch-pathognomische Deutungen vornimmt, demjenigen des Fräuleins von Sternheim diametral entgegengesetzt ist. ${ }^{52}$ Während Fräulein von Sternheim im ganz buchstäblichen Sinne nur Augen für die Tugend hat, ${ }^{53}$ vermutet Derby bei einer Röte im Gesicht prompt Liebschaften, wie sie dem höfischen Werteund Verhaltenskodex entsprechen würden.

Wie schnell Derbys pathognomische Deutungen dem Fräulein von Sternheim - ganz im Einklang mit dem ihm vertrauten semiotischen System der Hofkultur - nicht nur die Tugendhaftigkeit abzusprechen bereit sind, sondern in welch hohem Maße sie sich dabei als geradezu topisch erweisen, zeigt sich, als das Fräulein von Sternheim eine Frau T. kennen lernt, der sie Barmherzigkeit erweisen möchte:

Meine Sternheim sieht die Frau starr an, wird roth, nachdenklich, betrübt, alles schier in Einem Anblick [...]. [...] Unruhe nimmt sie ein [...]. Brennendroth und verwirrt eilt sie weg. Was T - dachte ich, muß das Mädchen mit dem Weibe haben; sie mag wohl irgend einmal Briefträgerinn, oder sonst eine dienstfertige

51 Ebd., S. 207f.

52 Zur Inkompatibilität der semiotischen Systeme von höfischer und bürgerlicher Kultur in La Roches Briefroman vgl. Alexandra Kleihues: Der empfindsame Blick.

53 Aufschlussreich ist Fräulein von Sternheims rhetorische Frage: »Hätte ich nicht selbst böse seyn müssen, wenn ich seine [Lord Derbys, Y.M.] Ränke hätte argwöhnen sollen?« (Sophie von La Roche: Geschichte des Fräuleins von Sternheim, Bd. 2, S. 284) 
Creatur in einem verborgenen Liebeshandel gewesen seyn. [...] $\mathrm{Hm}$ ! Hm! wie siehts mit dieser strengen Tugend aus? 54

Als Derby schließlich erfährt, dass nicht die Liebe zu einem anderen Mann, sondern die caritas ausschlaggebend für die beobachteten körperlichen Zeichen ist, äußert er sich zu seinem interpretatorischen Versagen wie folgt: "[I]ch fand, daß das Mädchen den wunderlichsten Charakter hat. Was T* wird sie roth und verwirrt, wenn sie etwas Gutes thun will [...]? $?^{55}$

Derby muss schließlich erkennen, dass das Fräulein von Sternheim eine andere "Gattung von Charakter ${ }^{56}$ darstellt als die ihm bislang bekannten Frauen. ${ }^{57}$ Seine Universalität beanspruchenden Kriterien der Physiognomik respektive Pathognomik erweisen sich nicht nur im Moment des Zusammenstoßes von höfischem und bürgerlichem Paradigma als unbrauchbar, sondern nicht zuletzt auch angesichts der Individualität einzelner Charaktere. Es zeigt sich, dass Gesichter sich eben nicht so einfach wie aufgeschlagene Bücher lesen lassen. ${ }^{58}$ Die "Buchstaben" des "göttlichen Alphabets", deren Dechiffrierung Lavater nur wenig später verheißen wird, entpuppen sich bereits als nicht allgemein applizierbar. ${ }^{59}$

\section{Epistolare Physiognomik und Pathognomik}

Die eminente Fehleranfälligkeit des Lesens von Gesichtern trifft allerdings nicht auch auf das Lesen von Briefen zu. ${ }^{60}$ So systematisch in der Geschichte

Ebd., Bd. 1, S. $210 f$.

Ebd., S. 215.

56 Ebd., S. 251.

57 Diesen Umstand resümiert Derby anlässlich eines Maskenballs wie folgt: »Bey einem Ball, wo beynahe alle Weibspersonen Coquetten, und auch die Besten von der Begierde zu gefallen eingenommen sind, hängt sie der Uebung der Wohlthätigkeit nach. Andre werden durch die Versammlung vieler Leute und den Lermen eines Festes, durch die Pracht der Kleider und Verzierungen betäubt, durch die Musik weichlich gemacht, und durch alles zusammen den Verführungen der Sinnlichkeit bloß gegeben, Sie wird auch gerührt, aber zum Mitleiden für die Armen [...].« (Ebd., S. 248f.)

58 Zur Zentralmetapher der Lesbarkeit vgl. Hans Blumenberg: Die Lesbarkeit der Welt, Frankfurt a.M. 1981, im Hinblick auf die Physiognomik bes. S. 199-213.

59 Alle Teilzitate: Johann Caspar Lavater: Physiognomische Fragmente, Bd. 1, Vorrede [unpag.].

60 So sie denn zuverlässig zugestellt werden. Wenn etwa Lord Derby durch Bestechung des Post-Offiziers Fräulein von Sternheims Post abfängt und diese nur selektiv weiterleitet oder von einem Billet des Fräuleins von Sternheim verständnisrelevante Passagen abreißt, sind selbstredend Missverständnisse vorprogrammiert. 
des Fräuleins von Sternheim die Fehleranfälligkeit der Körpersemiotik vorgeführt wird, so konsequent wird die Zuverlässigkeit einer charakterologischen Lektüre von Briefen dargestellt (»O Freund, was für eine Seele mahlt sich darinn! «61). Nicht zufällig ist die Titelfigur, das Fräulein von Sternheim, in ganz buchstäblichem Sinne das Kind eines Briefverkehrs, hat sich doch ihre Mutter in den Oberst von Sternheim verliebt, weil sie »in seinen Briefen die Vortrefflichkeit seines Geistes und Herzens bewundert «62 (und d.h.: physiognomisch gelesen) hat.

Nur zu bezeichnend ist, wenn Lord Rich, der seine Liebe in platonischen Bahnen belässt, am Romanende statt der Hand des Fräuleins von Sternheim ihre Briefe erbittet: »[E]rlauben Sie mir, [...] Sie um dieses Urbild Ihrer Empfindungen zu bitten; lassen Sie, meine englische Freundinn, mich diese Züge Ihrer Seele besitzen [...]. «63 Wenn er auch letztlich zu dem resignativen Schluss gelangt, dass Fräulein von Sternheims "schöne[ ] Briefe [...] nicht sie selbst « ${ }^{64}$ sind, so zeigt dies doch, wie weit die gegenseitige Substitution von Brief und Seele gezogen werden kann, ja, dass ein Brief nicht nur auf das Innenleben seines Verfassers oder seiner Verfasserin verweist, sondern es nahezu bis zur Verwechslung repräsentiert. ${ }^{65}$

Die Figuren machen im Zuge ihrer Brieflektüren die genaue Funktionsweise ihrer semiotischen Operationen nicht transparent. Gerade aber diese offenbare Absenz jeglicher Erklärungsbedürftigkeit zeigt, wie eindeutig und unmittelbar die Relation ist, die zwischen Briefen und dem sich darin zeigenden Innenleben vorausgesetzt wird. Dabei kommt Briefen nicht nur eine Evidenz-, sondern gar eine untrügliche Beweiskraft zu. So schreibt Lord Rich:

Es war die Seele, die Gesinnungen [...], die ich liebte. Ihre [Fräulein von Sternheims, Y.M.] Papiere, die sie in der vollen Aufrichtigkeit ihres Herzens schrieb, beweisen mir, daß sie das Beste mir schenkte, so in ihrer Gewalt war; wahre Hochachtung für meinen Character, wahres Vertrauen, zärtliche Wünsche für mein Glück. ${ }^{66}$

61 Sophie von La Roche: Geschichte des Fräuleins von Sternheim, Bd. 2, S. 282.

62 Ebd., Bd. 1, S. 6.

63 Ebd., Bd. 2, S. 286.

64 Ebd., S. 298.

65 Zur Medialisierung von Emotionen und Substitution von Körpern durch Briefverkehr im 18. Jahrhundert vgl. Albrecht Koschorke: Körperströme und Schriftverkehr.

66 Sophie von La Roche: Geschichte des Fräuleins von Sternheim, Bd. 2, S. 290f. [meine Hervorhebung, Y.M.]. 
Analog zu Gesichtern können Briefe im Verständnis von La Roches Protagonisten das Innenleben ihrer Verfasser gleichsam »zeigen«; 67 man kann es geradezu "sehen ${ }^{68}{ }^{68}$ Die sich im 18. Jahrhundert vollziehende Umcodierung von der Rhetorik zur Ausdrucksästhetik lässt nicht nur Stil und Charakter, sondern auch Stil und Physiognomik in enge Nähe rücken. ${ }^{69}$ Schon Sulzer weiß zu berichten, dass der Schreibstil "von dem besondern Charakter des Verfassers herrühret « ${ }^{70}$ und folglich die »Schattirungen des Styls «71 den »Physionomien der Menschen selbst« ${ }^{72}$ gleichen. Auch Karl Philipp Moritz denkt in seinen Vorlesungen über den Sty ${ }^{3}$ diesen als Charakterausdruck des Schreibenden.

Der Topos vom Stil als 'Ausdruck ‘ der Persönlichkeit, der freilich bereits Vorläufer in der antiken Rhetorik kennt (uc vir, sic oratio), löst die in der ersten Hälfte des 18. Jahrhunderts noch dominante - ebenfalls auf einen antiken Topos zurückgehende - Vorstellung vom Stil als 'Einkleidung ab, wird im Sinne menschlicher Individualität gefasst und mit der Physiognomik enggeführt. ${ }^{74}$ Ausgerechnet auf Lichtenberg geht wohl die Begriffsprägung von der Physiognomik des Stils zurück, die eine Reaktion auf die Fallibilität der Körpersemiotik darstellt, insofern dieser Spielart der Physiognomik eine gesicherte(re) Erkenntnisfunktion zugeschrieben wird. ${ }^{75}$

Eine Engführung von Stil und Charakter gilt nicht zuletzt für die Gattung des Briefes, die seit jeher dem Topos vom Spiegel der Seele unterstellt ist. ${ }^{76}$ Ein prominentes Beispiel bildet Gellerts wirkmächtiger Briefsteller des Jah-

67 Ebd., S. 284.

68 Ebd., Bd. 1, S. 367.

69 Lessing bringt dies in einem Aperçu auf den Punkt: "Jeder Mensch hat seinen eignen Stil, so wie seine eigne Nase [...]. « (Gotthold Ephraim Lessing: 2. Anti-Goeze, in: ders.: Werke und Briefe in 12 Bänden, Bd. 9: Werke 1778-1780, hg. von Klaus Bohnen und Arno Schilson, S. 149-184, hier S. 150)

70 Johann Georg Sulzer: Allgemeine Theorie der schönen Künste, Lemma: Schreibart; Styl. (Schöne Künste.), S. 1047-1055, hier S. 1048.

71 Ebd., S. 1051.

72 Ebd.

73 Karl Philipp Moritz: Vorlesungen über den Styl oder praktische Anweisung zu einer guten Schreibart in Beispielen aus den vorzüglichsten Schriftstellern, 2 Bde., Berlin 1793-1794.

74 Einen historischen Überblick liefert Wolfgang G. Müller: Topik des Stilbegriffs. Zur Geschichte des Stilverständnisses von der Antike zur Gegenwart, Darmstadt 1981, bes. S. 99-108. Vgl. ebd., S. 101f.

76 Vgl. Wolfgang G. Müller: Der Brief als Spiegel der Seele. Zur Geschichte eines Topos der Epistolartheorie von der Antike bis Samuel Richardson, in: Antike und Abendland 26/2 (1980), S. 138-157. 
res 1751, der eine Ästhetik der Natürlichkeit mit einer charakterologischen Lektüre von Briefen verfugt. Gellert postuliert, dass man »durch seine Briefe seinen Character entdecken muß ${ }^{77}{ }^{77} \mathrm{ja}$, dass "Briefe wider unsern Willen Verräther unsers Verstandes, und oft unsers ganzen Charakters sind; [...] daß sie Beweise sind, ob es dunkel oder helle, ordentlich oder unordentlich, gesund oder krank in unserm Geiste aussieht. «"

Die innerhalb der Geschichte des Fräuleins von Sternheims vorgeführte Physiognomik des Briefstils mag sich dabei durchaus als Lektüre-Muster für das reale Lesepublikum verstanden wissen, das seine eigenen Charakter-Urteile fällt. Nicht zufällig bestimmt Adelung das Publikum ausgehend von seiner Funktion als »richtende[s] Publicum " ${ }^{79}$ Auch »die Leser eines Schriftstellers" werden, so Adelung, »dessen Publicum genannt, ob sie gleich nirgends im Ganzen versammelt sind, ihr Ausspruch auch nirgends im Ganzen gehöret wird «.80

Nun kann die reale Leserschaft des Briefromans, die von seinen fiktiven Protagonisten ontologisch getrennt ist, weder die zur Verhandlung stehenden Körper realiter sehen noch findet sie im Briefroman Detailbeschreibungen des Äußeren, die der Einbildungskraft als Ausgangs- oder Anhaltspunkt dienen könnten, und findet sich so auf physiognomische Stillektüren zurückgeworfen - aber wohlgemerkt auch auf pathognomische. So ließe sich in der Geschichte des Fräuleins von Sternheim eine Satzzeichenmaximierung im Tagebuch der Madam Leidens - eine Flut an Gedankenstrichen, Frage- und Ausrufungszeichen, die syntaktische Notwendigkeiten übersteigt - bereits im Medium des Visuellen und damit unabhängig von einer propositionalen Brieflektüre als Anzeichen von Affektregungen deuten; ${ }^{81}$ sie »kann zum Beweis dienen, wie sehr ein heftiger Schmerz des Gemüths das edelste Herz zerrütten kann«. ${ }^{82}$ Dass Satzzeichen sich pathognomisch deuten lassen, erfährt gar eine Kodifikation in Adelungs Vollständiger Anweisung zur Deutschen

77 Christian Fürchtegott Gellert: Briefe, nebst einer praktischen Abhandlung von dem guten Geschmacke in Briefen, Leipzig 1751, S. 118.

78 Ebd., S. 120f. [meine Hervorhebung, Y.M.]. Freilich nimmt Gellert dies zum Anlass, auf "mehr Mühe um die Schreibart« (ebd., S. 121) zu insistieren.

79 Johann Christoph Adelung: Grammatisch-kritisches Wörterbuch, Bd. 3, Leipzig 1798, Lemma: Das Publicum, S. 856-857, hier S. 857.

80 Ebd.

81 Zum Ausdrucksgehalt von Satzzeichen und ihrer Schriftbildlichkeit vgl. Andrea Polaschegg: Ausdruckskunst! Satzzeichen als Indizien des Affekts in Ode und Briefroman des 18. Jahrhunderts, in: Die Poesie der Zeichensetzung. Studien zur Stilistik der Interpunktion, hg. von Alexander Nebrig und Carlos Spoerhase, Bern 2012, S. 157-182. 
Orthographie, die das Frage- und das Ausrufungszeichen als "Zeichen der Gemüthsstellung" und treffender noch als "Affect-Zeichen" klassifiziert. ${ }^{83}$ Von dem Gedankenstrich weiß Adelung zu berichten, dass er »[b]esonders in einem heftigen Affecte, der immer die Abgebrochenheit und in derselben starke Pausen liebt« ${ }^{84}$ Anwendung finde.

Während jedoch eine Satzzeichenpoetik ein sich schriftbildlich manifestierendes Phänomen darstellt (nicht zufällig wird Adorno die druckgrafisch beobachtbaren Satzzeichen als Physiognomie eines Textes bezeichnen ${ }^{85}$ ), sind stilistische Merkmale wie etwa Tropen oder syntaktische Besonderheiten zwar interpretierbar, jedoch nicht sauf einen Blick ‘ mit den Augen erfassbar. Physiognomik und Pathognomik, verstanden als gestaltdeutende Kulturtechniken, münden spätestens dann in Aporien, wenn nicht mehr von distinkten stilistischen Charakteristika, sondern vom Abstraktum 'Stik als Ganzem die Rede ist. Ein Abstraktum ist allenfalls in figuralem Sinne sichtbar`. Hier hat sich die Physiognomik nicht nur der Visualität entledigt, sondern auch ihrer Bindung an eine, ob nun reale oder imaginierte körperliche Gestalt. Wer einem Stil etwas ransieht $<$, vollzieht schlechterdings eine andere mentale Operation als jemand, dessen Blick sich auf einen Körper richtet, wie auch immer dieser im Einzelnen geartet sein mag. Paradoxerweise aber schmälert die problematische 'Sichtbarkeit des Stils in keiner Weise seine physiognomische Evidenzkraft, sondern es ist gerade die Physiognomik des Stils, der gegenüber der Fallibilität der Körpersemiotik und den Grenzen des Sinnesorgans >Auge in La Roches Briefroman epistemologisch der Vorzug gegeben wird.

83 Vgl. Johann Christoph Adelung: Vollständige Anweisung zur Deutschen Orthographie, nebst einem kleinen Wörterbuche für die Aussprache, Orthographie, Biegung und Ableitung, Leipzig 1788, S. 363-371; S. 395-398. Adelung freilich plädiert für einen maßvollen Satzzeichengebrauch (vgl. ebd., S. 393-398).

84 Ebd., S. 390.

85 Vgl. Theodor W. Adorno: Satzzeichen [1956], in: ders.: Gesammelte Schriften in 20 Bänden, hg. von Rolf Tiedemann unter Mitwirkung von Gretel Adorno, Susan Buck-Morss und Klaus Schultz, Bd. 11: Noten zur Literatur, Frankfurt a.M. 2003, S. 106-113, bes. S. 106. 